\title{
Layered Earth Propagation in the Vicinity of Point Barrow, Alaska
}

\author{
Glenn M. Stanley²
}

(September 1, 1959)

\begin{abstract}
The relative field strength of a vertically polarized low-frequency radio signal was measured as a function of distance over several radial paths in the vicinity of Point Barrow, Alaska. The attenuation of the recorded signal was very much less than predicted by the theory of propagation of a ground wave signal over a plane, homogeneous, infinitely conducting earth. The analysis of these data in terms of a plane, layered, finitely conducting earth appears to resolve the anomaly.
\end{abstract}

\section{Introduction}

For several years, studies of effective ground conductivities in Alaska have been in progress by the Geophysical Institute at the University of Alaska. The project has been carried on under the auspices of the U.S. Navy and the Arctic Institute of North America. The data used in this paper were gathered under a Buships contract administered by the Signal Corps.

The relative field strength, as a function of distance from the transmitting antenna, was measured along several radials from each of 25 Civil Aeronautics Administration radio ranges and beacons in Alaska. These ranges and beacons operate in the frequency range of from 200 to $400 \mathrm{kc}$. The measurements were made from a low-flying aircraft equipped with a sensitive receiver, an EsterlineAngus recorder, and a nearly vertical trailing wire antenna. The effective ground conductivity is obtained by a comparison of the experimental relative field strength values with families of theoretical curves obtained by Norton's method. From this comparison, the value of conductivity that best fits the experimental data is taken as the value of effective conductivity over the path.

Signals were recorded continuously along radii from the transmitting antennas for distances up to 120 miles. Check points along the flight paths were obtained from U.S.G.S. 1:250,000 topographical contour maps. The aircraft used was equipped with a gyrocompass, and constant course checks were made with maps. In most instances, deviations from the radial course were less than a few hundred feet.

As all signals were recorded along radii from the transmitting antenna, no corrections were necessary for assymetrical transmitting and receiving antennas. At a distance of approximately 30 wavelengths, no appreciable change in the signal level was apparent in a $1 / 2$ mile horizontal deviation from the radial

1 This paper was presented at the Symposium on VLF Radio Waves held in Boulder, Colo., January 1957 (invited paper).

2 Present address: Arctic Aeromedical Laboratory, APO 731, Seattle, Wash. flight path. In addition, no appreciable changes in the signal levels were noted for changes of a few hundred feet in elevation at the same distance. The elevation of the receiving antenna, however, was kept to within $400 \mathrm{ft}$ of the local terrain (and in general, was nearer to $200 \mathrm{ft}$ ) to reduce the effect of interference between the direct and the reflected signal.

Relative field strengths were used throughout the computations of effective conductivity and no attempt was made to measure absolute field strength.

Measurements made in the vicinity of Point Barrow, Alaska, showed that the signals did not attenuate as rapidly as might have been expected for a region known to be underlain with permafrost to depths of 500 to $1,000 \mathrm{ft}$. Flight runs were made from Point Barrow in June and again in August. With one exception, the signal strength decreased less rapidly than predicted by the theory of a plane homogeneous earth for an infinitely conducting ground.

\section{Theoretical Consideration}

To make clear the implications of the above mentioned anomaly, it is appropriate to review briefly the theory underlying the experimental measurements. The surface wave field strength at short distances is given by the relation

$$
E=2 E_{o}\left|F_{e}\right| \text { and } E_{o}=\frac{\text { const. }}{\rho} \text {, }
$$

where $E_{o}=$ the maximum field strength of the antenna if it were located in free space

$\rho=$ the distance from the transmitting antenna to the receiving antenna and

$\left|F_{e}\right|=$ the surface wave attenuation factor;

provided the antennas are very near the ground and that

$$
2 \lambda<\rho<\frac{50}{f^{1 / 3} \mathrm{mc}} \mathrm{mi} .
$$


The surface wave attenuation factor may be obtained by methods given by Norton [1]. ${ }^{3}$

Taking the ratio of the relative field strengths at two points at distance $\rho_{n}$ and $\rho_{x}$ respectively, from the transmitting antenna, where $\rho_{n}$ is greater than $\rho_{x}$, it may be seen that

$$
\frac{E_{n}}{E_{x}}=\frac{\left|F_{e}\right|_{n} \rho_{x}}{\left|F_{e}\right|_{x} \rho_{n}}
$$

Using this relation a relative field strength curve may be drawn from any convenient initial point. Each set of values of ground constants will give one such curve. Figure 1 shows a family of these curves for $f=236 \mathrm{kc}, \epsilon=5 \epsilon_{o}$ and an initial point of $2.3 \mathrm{mi}$ from the transmitting antenna.

The surface wave attenuation factor $F_{e}$ is given by

$$
F_{e}=1-i\left(\pi p_{e}\right)^{1 ! 2} e^{-p} \operatorname{erfc}\left(i p_{e}^{1 / 2}\right),
$$

where $p_{e}$ is the effective numerical distance and is given by

$$
p_{e}=\left|p_{e}\right| e^{i b}
$$

Assuming a homogeneous earth, $b$ is negative and $\left|F_{e}\right|$ is less than unity. However, Wait has shown that (for certain conditions of horizontally stratified media) $b$ may be positive and $\left|F_{e}\right|$ greater than unity $[2,3,4]$. Wait presents tables of $\left|F_{e}\right|$ as a function of $\left|p_{e}\right|$ for positive values of $b$ to +75 degrees and for negative values of $b$ to -90 degrees. The negative values were obtained using the results of Norton. $\left|p_{e}\right|$ can be computed in terms of the impedance of the surface of the top layer looking normally downward. The impedance of the top layer is related to the impedance of each succeeding lower layer by a recurrence equation. For a twolayer earth, where $\sigma_{2}>>\sigma_{1}$, and $h_{1}$ is not too small,

$$
\begin{aligned}
\left|p_{e}\right| & =\frac{\pi \rho \mu \omega}{(120 \pi)^{2} \lambda_{0} \sigma_{1}}\left(A^{2}+B^{2}\right) ; \\
b & =\tan ^{-1}\left(\frac{2 A B}{A^{2}-B^{2}}\right) ;
\end{aligned}
$$

where

$$
\begin{aligned}
& A=\frac{\tanh w \sec ^{2} w}{1+\tanh ^{2} w \tan ^{2} w} ; \\
& B=\frac{\tan w \operatorname{sech}^{2} w}{1+\tanh ^{2} w \tan ^{2} w}
\end{aligned}
$$

and

$$
w=\frac{\left(\sigma_{1} \mu w\right)^{\frac{1}{2}} h_{1}}{\sqrt{2}}
$$

${ }^{3}$ Figures in brackets indicate the literature references at the end of th is paper
From these relations the effective numerical distance $\left|p_{e}\right|$, and the phase angle $b$, may be obtained for a given frequency, a given thickness and conductivity of the upper layer. When $\left|p_{e}\right|$ and $b$ are known, the surface wave attenuation factor $\left|F_{e}\right|$ can be determined. Since $\left|F_{e}\right|$ is the only unknown entering the equation for the relative field strength, curves may then be drawn for different values of the parameters $\sigma_{1}$ and $h_{1}$. Figure 2 shows curves of relative field strength for some values of $h_{1}$ taking $\sigma_{1}$ to be constant and equal to $1 \mathrm{mmho} / \mathrm{m}$.

\section{Results and Discussion}

Returning to the experimental values of relative field strength obtained near Point Barrow, an attempt is made to explain the low attenuation of the signals in terms of layered earth propagation.

The existence of highly conducting saline layers or lenses at depths of from 10 to $100 \mathrm{ft}$ in the immediate vicinity of Point Barrow is deduced from test wells driven by the Arctic Contractors during the course of their work on U.S. Navy Petroleum Reserve \#4. The extent and exact nature of these layers or lenses is not known. However, relative field strength measurements indicate that the propagation characteristics change markedly at distances of from 60 to $100 \mathrm{mi}$ southward from Point Barrow. In addition, there is visual evidence of an abandoned beach line at about this same location. Mr. Max Brewer, of the Arctic Research Laboratory at Point Barrow reports that the salinity of these layers near Point Barrow is equal to, or greater than that of sea water.

Figure 3 shows flight runs 93-95 made in June 1954. The vertical lines depict the spread of the three runs while the circles represent average values. Other flight runs in the vicinity have shown similar conditions of anomalous propagation.

Thus, it is reasonable to approximate the ground in the vicinity of Point Barrow by a two layer ground. The conductivity of the lower layer has been assumed to be equal to that of sea water $(5000 \mathrm{mmho} / \mathrm{m})$. Several families of relative field strength curves were drawn for different values of the conductivity of the upper layer $\sigma_{1}$, and for different thicknesses of the upper layer $h_{1}$. The thickness of the lower layer was assumed to be semi-infinite. Figure 4 shows the average values obtained from flight runs 89-92 and 202-205 superimposed on the relative field strength curves for $\sigma_{1}$ equal to $10 \mathrm{mmho} / \mathrm{m}$. The comparison of the experimental curve with the theoretical curves suggests that the apparent thickness of the upper layer $h_{1}$ is equal to approximately $9 \mathrm{~m}$.

Flight runs 93-95 and 197-199, which were about $30 \mathrm{deg}$ to the East, and flight runs 193-196, approximately $30 \mathrm{deg}$ to the West of those shown in figure 2 have similar characteristics. In contrast, flight runs $85-88$, show a very high signal level. No combination of ground constants that has been tried will produce a relative field strength curve which matches the experimental curve corresponding to this high signal level. 

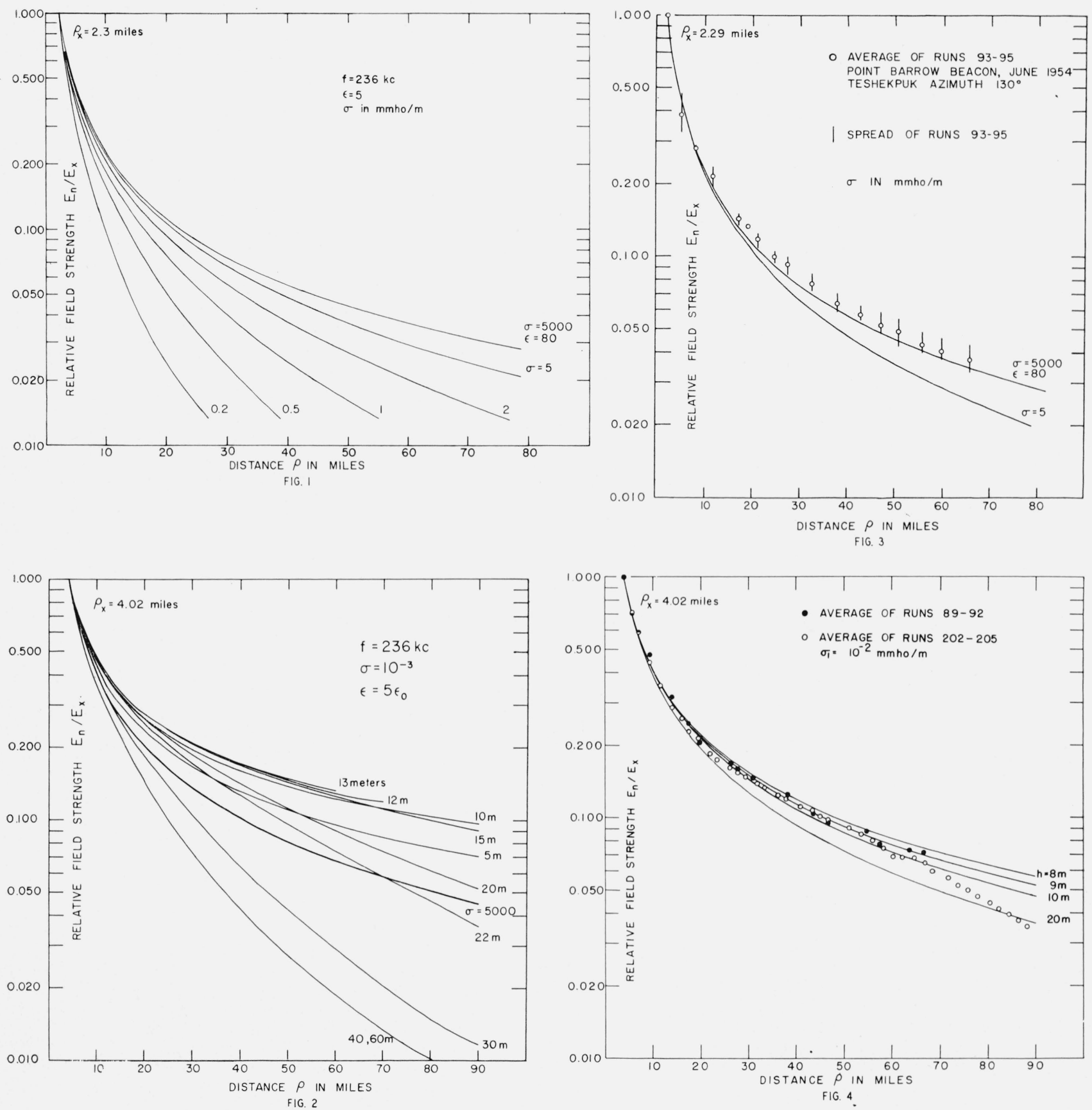

Figures 1, 2, 3, 4. Field strength versus distance curves.

The analysis of these data indicates, that at a frequency of $236 \mathrm{kc}$, there is, indeed, layered earth propagation in the vicinity of Point Barrow, Alaska. The analysis further suggests that the ground conductivity of the upper $9 \mathrm{~m}$ is approximately 10 $\mathrm{mmho} / \mathrm{m}$, when measured by the method outlined.

The area of measurement which encompasses most of the Arctic slope is essentially flat and free from manmade interference such as fences, railroads, powerlines, and other sources of disturbance. It therefore appears that this area would provide an excellent test site for various low-frequency propagation studies.

\section{References}

[1] K. A. Norton, The calculation of ground-wave field intensity over a finitely conducting spherical earth, Proc. IRE, 29, 623 (1941).

[2] James R. Wait, Radiation from a vertical electric dipole over a stratifield ground, Trans. IRE PGAP, AP-1, 9 (1953).

[3] James R. Wait and W. C. G. Fraser, Radiation from a vertical dipole over a stratified ground (part II), Trans. IRE PGAP, AP-3, 144 (1954).

[4] James R. Wait, The theory of electromagnetic surface waves of geological conductors, Geofis. pura e appl. (Milano), 28, 47 (1954).

Boulder, Colo.

(Paper 64D1-43) 